\title{
Second-line chemotherapy for gastric cancer: a new issue lies ahead in global trials
}

\author{
Hiroya Takiuchi
}

Received: 25 January 2011/ Accepted: 13 June 2011/Published online: 23 July 2011

(c) The International Gastric Cancer Association and The Japanese Gastric Cancer Association 2011

\begin{abstract}
Chemotherapy for gastric cancer has been advancing fairly well. It has been indicated that not only advances in first-line chemotherapy but also those in second-line chemotherapy have contributed to the prolongation of overall survival. The Arbeitsgemeinschaft Internistische Onkologie (AIO) study supports the idea that second-line chemotherapy is appropriate in patients with a good general condition. Also, the Japan Clinical Oncology Group (JCOG) integral analysis suggests that advances have been made in second-line chemotherapy. However, most recently reported studies of second-line chemotherapy have been conducted as small-scale phase II or retrospective trials. No randomized control trial to establish standard treatment has been reported. Which regimen is the most appropriate as second-line therapy must be investigated in the future. Currently, molecularly targeted agents for gastric cancer are being developed and tested in global trials. As a new issue in global trials, second-line chemotherapy has been emphasized. In recent global trials, subset analysis showed regional differences in overall survival. This was possibly associated with the regional differences in second-line chemotherapy. When developing new molecularly targeted agents for first-line chemotherapy, we cannot ignore the result that the proportion of patients in whom treatment was switched to second-line chemotherapy was high in Asia. In planning a global trial, this new issue should be sufficiently discussed.
\end{abstract}

Keywords Gastric cancer - Second-line chemotherapy · Global trial $\cdot$ Molecularly targeted agent

H. Takiuchi $(\bowtie)$

Cancer Chemotherapy Center, Osaka Medical College,

2-7 Daigaku-Cho, Takatsuki, Osaka 569-8686, Japan

e-mail: in2028@poh.osaka-med.ac.jp

\section{Introduction}

Gastric cancer is frequent in Asia, South America, and Eastern Europe, accounting for more than 800,000 new cases per year worldwide, and it is the second most common cause of cancer death [1]. Because early detection strategies are rarely practiced, except in Japan and Korea, most patients will present with advanced-stage disease, and will therefore need palliative chemotherapy. Some chemotherapy regimens have been established as first-line therapy, and some progress has been made in the treatment of advanced-stage disease [2-12]. However, almost all patients with metastatic gastric cancer will develop progressive disease (PD) after first-line therapy. With the availability of several active chemotherapy drugs, many patients who retain a good performance status after the initial treatment remain good candidates for additional therapy.

However, most clinical studies of second-line chemotherapy have been conducted as phase II, small-scale trials. The data obtained are limited, and there is no standard second-line chemotherapy. In this review, differing from previous reviews of second-line chemotherapy [13, 14], I have clarified the significance of second-line chemotherapy based on the recently reported results of randomized control trials of first-line chemotherapy. On the other hand, I refer to the concept of second-line chemotherapy as a potentially confounding factor in recent global trials.

\section{Evidence for second-line chemotherapy}

Chemotherapy for advanced/recurrent gastric cancer has been advancing fairly well. As evidence, the median survival in recent randomized comparative studies involving 
patients with advanced/recurrent gastric cancer was markedly longer than that in previous studies [2-12]. It was indicated that not only advances in first-line chemotherapy but also advances in second-line chemotherapy contributed to the prolongation of survival. However, no phase III study has verified the significance of second-line chemotherapy. The results of the Arbeitsgemeinschaft Internistische Onkologie (AIO) comparative study suggest its significance; this study was reported at the 2009 annual meeting of the American Society of Clinical Oncology (ASCO) [15]. In this study, patients in whom first-line therapy led to progressive disease were divided into 2 groups: best supportive care (BSC) and irinotecan groups, to evaluate the usefulness of irinotecan in second-line therapy. In regard to the statistical background, 60 patients per group (2 groups: 120 patients) were required, assuming that irinotecan administration may prolong the median survival time (MST) from 2.5 to 4 months, with an $\alpha$ error (paired) of 5\% and a detection power of $80 \%$. However, case registration was insufficient, and the clinical study was completed when 40 patients were enrolled in each of the two groups. The results of analysis were reported. In the irinotecan group, the response rate was $0 \%$. However, the stable disease rate was $58 \%$, and improvement of tumor-related symptoms was achieved in $44 \%$ of the patients. In addition, the MSTs in the irinotecan and BSC groups were 123 and 76 days, respectively. Statistically, the overall survival (OS) was longer in the irinotecan group $(p=0.0027)$. These results support the idea that secondline chemotherapy is appropriate in patients with a good general condition. However, which regimen is the most appropriate as second-line therapy must be investigated in the future.

\section{Significance of second-line chemotherapy with respect to randomized comparative studies in Japan}

The JCOG 9205 study was started by the Japan Clinical Oncology Group (JCOG) in 1992. Initially, 3 groups, 5-fluorouracil (5-FU), 5-FU + cisplatin, and uracil and tegafur (UFT) + mitomycin C (MMC) groups, were compared [4]. However, the mid-analysis results suggested that UFT + MMC therapy may be less potent than 5-FU therapy. After mid-analysis, the UFT + MMC group was excluded from the subject cohort. Finally, in this study, the results were compared between the 5-FU and 5 -FU + cisplatin groups. The OS in the $5-\mathrm{FU}+$ cisplatin group did not exceed that in the 5-FU group. The MST for monotherapy with 5-FU was 7.1 months, and the median progression-free survival (PFS) was 1.9 months. In the JCOG 9912 study, which was conducted subsequently, monotherapy with 5-FU was additionally employed as a control regimen [10]. The MST for monotherapy with 5-FU was 10.8 months, and the median PFS was 2.9 months. Survival data regarding monotherapy with 5-FU, involving different time-related background factors, were obtained in the two randomized comparative studies conducted by the same clinical study group (Table 1). The JCOG performed integral analysis regarding the two studies, focusing on second-line chemotherapy, and reported the results at the ASCO 2010 meeting [16]. To harmonize the inclusion criteria in the two studies, patients with intestinal stenosis in the JCOG 9205 study and those with adjuvant chemotherapy in the JCOG 9912 study were excluded. Overall survival, time to treatment failure (TTF), and OS minus TTF (OS-TTF) were compared after adjusting for baseline factors using the Cox proportional hazard model. Interestingly, the MST after second-line therapy in the 5-FU group was longer in the JCOG 9912 study.

There are two reasons for the above finding: firstly, the number of effective agents available for second-line therapy in the JCOG 9912 study was larger than the number available at the time of the JCOG 9205 study. In the JCOG 9205 study, early-generation drugs such as cisplatin and MMC were used for second-line therapy. On the other hand, in the JCOG 9912 study, newer drugs such as a taxane and irinotecan were primarily employed for second-line therapy; irinotecan- or taxane-containing regimens were selected in $9 \%(8 / 94)$ of the subjects in the JCOG 9205 study and in 67\% (157/233) in the JCOG 9912 study. The difference in treatment options for second-line chemotherapy may have contributed to an MST difference of 3.7 months. On the other hand, the proportion of patients in the 5-FU group in whom treatment was switched to second-line chemotherapy should be compared between the two studies. In approximately $52 \%$ of patients receiving 5-FU alone in the JCOG 9205 study, treatment was switched to second-line chemotherapy. In the JCOG 9912 study, the percentage was approximately $83 \%$, showing a $31 \%$ increase. This difference may also have led to the MST difference of 3.7 months. Even after adjusting for baseline factors, TTF was similar in the two studies; however, both OS and OS-TTF were longer in the JCOG 9912 study than in the JCOG 9205 study. It was concluded

Table 1 Differences of efficacy profiles and second-line treatment in the 5-fluorouracil arms between the JCOG 9205 and JCOG 9912 trials

\begin{tabular}{lclll}
\hline & $\begin{array}{l}\text { ORR } \\
(\%)\end{array}$ & $\begin{array}{l}\text { PFS } \\
\text { (months) }\end{array}$ & $\begin{array}{l}\text { MST } \\
\text { (months) }\end{array}$ & $\begin{array}{l}\text { Second-line } \\
\text { treatment (\%) }\end{array}$ \\
\hline JCOG 9205 trial [4] & 9 & 1.9 & 7.1 & 52 \\
JCOG 9912 trial [10] & 11 & 2.9 & 10.8 & 83 \\
\hline
\end{tabular}

JCOG Japan Clinical Oncology Group, ORR overall response rate, $P F S$ progression-free survival, MST median survival time 
that survival after treatment failure of 5-FU alone was longer in the JCOG 9912 study even when some potential confounding factors were adjusted for. The results of this combined analysis suggest that advances have been made in second-line chemotherapy and support the use of second-line chemotherapy for gastric cancer. Physicians likely play a key role in whether or not patients receive secondline chemotherapy. Unfortunately, we currently have little evidence to guide treatment. I recommend that patients and physicians earnestly discuss the risks and benefits of second-line chemotherapy using the current best evidence on tolerability and effectiveness.

\section{Regional differences in second-line chemotherapy and new issues in global trials}

Trials of the same regimen, S-1 plus cisplatin, were conducted in Japan and other countries. When comparing the SPIRITS trial (S-1 vs. S-1 plus cisplatin), which was carried out in Japan, with the FLAGS trial (5-FU plus cisplatin vs. S-1 plus cisplatin), which was conducted as a global study, there was a regional difference in second-line chemotherapy; there was a marked difference in the proportion of patients in whom treatment was switched to second-line chemotherapy between the two trials $[9,11]$. The proportion of patients in whom treatment was switched to second-line chemotherapy was $73 \%$ in the SPIRITS study in Japan, whereas it was only $31 \%$ in the FLAGS trial. Such a low percentage was also common in other recently reported global studies. The second-line chemotherapy rates ranged from 70 to $83 \%$ in studies conducted in Japan, including the JCOG 9912 study [10-12], whereas the rate was only $15 \%$ in the REAL-2 trial involving the United Kingdom [7]. As a background factor, we must consider that the insurance coverage systems in Japan and other countries differ markedly. In particular, health insurance in the United Kingdom does not cover second-line chemotherapy; therefore, first-line chemotherapy is very important. The median survival in a phase III study recently reported in Japan was 2-3 months longer than that reported in Europe and the United States [7-12]. This finding may be associated with the difference in the proportion of patients in whom treatment was switched to second-line chemotherapy.

Currently, molecularly targeted agents for gastric cancer are being developed primarily in Japan and Korea and are being tested in global trials. As a new issue in these global trials, second-line chemotherapy has been emphasized. The ToGA study, in which Japanese and Korean patients accounted for more than $50 \%$ of the subjects, investigated the efficacy of first-line chemotherapy with trastuzumab in human epidermal growth factor receptor 2 (HER2)-positive advanced gastric cancer patients; 584 patients meeting eligibility criteria were randomly assigned to receive 5-FU or capecitabine + cisplatin (FC group: $n=290$ ), or 5-FU or capecitabine + cisplatin + trastuzumab $\quad(\mathrm{FC}+\mathrm{T}$ group: $n=294$ ) therapies. The median survival in the $\mathrm{FC}+\mathrm{T}$ group (13.8 months) was significantly longer than that in the FC group (11.1 months) $(p=0.0046)$, suggesting the usefulness of trastuzumab in HER2-positive gastric cancer patients [17]. In this study, subset analysis showed regional differences in survival; trastuzumab did not influence survival in Asia, but markedly influenced survival in South America. This finding was possibly related to regional differences in second-line chemotherapy, as described above. Approximately $50 \%$ of the subjects consisted of Korean and Japanese patients. In these two countries, second-line chemotherapy is positively performed in clinical practice. On the other hand, in South America, second-line chemotherapy is rarely performed. Therefore, the influence of first-line chemotherapy; that is, that of trastuzumab, may have been more marked in South America.

Similarly, in the AVAGAST trial reported at the ASCO 2010 meeting, there were also differences in the proportions of patients in whom treatment was switched to second-line chemotherapy [18]. In that study, there was no influence of bevacizumab on survival in Asia, similar to the lack of influence of trastuzumab in the ToGA trial. In PanAmerica, bevacizumab markedly influenced survival. This finding was possibly associated with regional differences in second-line chemotherapy (Table 2). In Asia, the proportion of patients in whom treatment was switched to secondline chemotherapy was high, $66 \%$, whereas the values were 31 and 21\% in Europe and Pan-America involving South America, respectively. Briefly, the influence of first-line chemotherapy on survival may be very marked in areas other than Asia. However, when many Japanese/Korean patients are registered, survival after second-line chemotherapy may be prolonged; therefore, there may be no significant difference in the OS. In the future, when developing molecularly targeted agents for first-line chemotherapy, we cannot ignore that there are regional differences in second-line chemotherapy. In planning global trials in the future, this issue should be sufficiently discussed.

Table 2 Proportions of patients receiving second-line chemotherapy by region in the AVAGAST trial [18]

\begin{tabular}{llll}
\hline Region & $\begin{array}{l}\text { Patients } \\
\text { entered }(n)\end{array}$ & $\begin{array}{l}\text { Patients receiving } \\
\text { second-line treatment }(n)\end{array}$ & $\%$ \\
\hline Asia & 376 & 248 & 66 \\
Europe & 249 & 78 & 31 \\
Pan-America & 149 & 32 & 21 \\
\hline
\end{tabular}




\section{Present status and future directions of second-line chemotherapy}

Most recently reported studies of second-line chemotherapy consist of small-scale phase II or retrospective trials [19-33]. No randomized control trial to establish standard treatment has been conducted. In clinical practice, irinotecan, docetaxel, or paclitaxel is selected in most patients. However, the effects of monotherapy are limited [20-25]. Various combination therapies have been investigated in small-scale, phase II studies [26-33]. However, according to the results of some recent studies, the response rates ranged from approximately 10 to $20 \%$, and PFS ranged from 2.5 to 4.0 months. There may be no marked differences among these combination therapies (Table 3). One study reported a median survival of 12 months. However, this may have depended on patient selection. As of now, that is all the information we can share. At the time of this writing, I think monotherapy is a reasonable option as a second-line treatment, and combination strategies should be used as a fall-back position. In Japan, weekly paclitaxel is widely used as the second-line chemotherapy in daily clinical practice. On the other hand, the AIO comparative study supported the use of irinotecan for second-line chemotherapy [15]. Much debate has focused on whether irinotecan or weekly paclitaxel is the better second-line agent. Among randomized control trials of second-line chemotherapy that are being conducted, "a randomized phase III study of irinotecan versus weekly paclitaxel in unresectable or recurrent gastric cancer refractory to combination therapy of fluorouracil plus platinum (WJOG 4007G)", has been carried out by the West Japan Oncology Group (WJOG). In this study, the primary endpoint was overall survival. Secondary endpoints were PFS, adverse events, and the response rate in patients with target lesions. The sample size was 220 in total, which allowed for the detection of irinotecan superiority over weekly paclitaxel in terms of OS. Final analysis will be performed in 2011. These study results are very important. It should be clarified which of the two agents, irinotecan or paclitaxel, is appropriate as a biologic, platform agent for second-line chemotherapy, and whether the effects of the two agents are similar.

Currently, several second-line or subsequent molecularly targeted agents are being developed and tested in global studies (Table 4). A randomized control trial of lapatinib involving HER2-positive gastric cancer patients (TYTAN trial) is being conducted (weekly paclitaxel vs. weekly paclitaxel + lapatinib). Furthermore, a randomized control trial of a mammalian target of rapamycin (mTOR) inhibitor, everolimus, for BSC is being performed in patients receiving second- and third-line therapies (GRANITE-1 trial) [34]. For new drug development, global trials are also necessary in the future. However, in randomized control trials in which OS is established as the primary endpoint of first-line chemotherapy, it is difficult to detect a difference unless molecularly targeted agents with a clear target, such as trastuzumab, are employed; this difficulty arises because there are regional differences in second-line chemotherapy. In particular, Japan and Korea,
Table 3 Efficacy profiles of combination chemotherapy in the second-line setting

ORR overall response rate, $P F S$ progression-free survival, $M S T$ median survival time, $N E$ not evaluated

\begin{tabular}{lllll}
\hline Regimen & ORR (\%) & PFS (months) & MST (months) & Reference number \\
\hline Paclitaxel/doxifluridine & 18.2 & 4.0 & 10.7 & {$[26]$} \\
Paclitaxel/capecitabine & 34.6 & 4.5 & 7.5 & {$[27]$} \\
Docetaxel/doxifluridine & 18.8 & 2.6 & 12.7 & {$[28]$} \\
Docetaxel/irinotecan & 20.4 & 2.7 & 8.9 & {$[29]$} \\
Docetaxel/oxaliplatin & 10.5 & 4.0 & 8.1 & {$[30]$} \\
Irinotecan/5-fluorouracil & 18.2 & 2.3 & 5.1 & {$[31]$} \\
Irinotecan/capecitabine & 17.0 & 3.1 & 6.5 & {$[32]$} \\
Methotrexate/5-fluorouracil & 9.0 & NE & 7.9 & {$[33]$} \\
\hline
\end{tabular}

Table 4 Phase III studies of targeted agents for second-line treatment in advanced gastric cancer

\begin{tabular}{llllll}
\hline Agent & Target & Chemotherapy partner & $N$ & Endpoint & Status \\
\hline Lapatinib & HER2 EGFR & Paclitaxel & 260 & OS & Ongoing \\
Ramucirumab & VEGFR-2 & Paclitaxel & 663 & OS & Ongoing \\
Everolimus & mTOR & None & 442 & OS & Ongoing \\
\hline
\end{tabular}

$O S$ overall survival, $m T O R$ mammalian target of rapamycin, HER2 human epidermal growth factor receptor 2, EGFR epidermal growth factor receptor, $V E G F R$-2 vascular endothelial growth factor receptor 2 
where second-line chemotherapy is actively performed, play a principal role in registration. For the future development of molecularly targeted agents, it might be necessary to discuss the adoption of PFS as the primary endpoint.

\section{Conclusions}

At this time, no standard second-line chemotherapy has clearly emerged in gastric cancer treatment, and none of the new molecularly targeted agents under investigation has been identified as being appreciably useful for secondline-chemotherapy. Given the lack of solid evidence, it is too early to know whether a number of novel regimens will ultimately achieve traction as useful standard second-line chemotherapies. New evidence and new drugs are needed to make the necessary further improvements in the management of gastric cancer. In global trials, however, we have learned of the difficulties in selecting survival benefit as the primary endpoint, with these difficulties arising because of the regional differences in the management of this disease. In planning global trials, this new issue should be sufficiently discussed.

\section{References}

1. Jemal A, Siegel R, Ward E, Murray T, Xu J, Smigal C, et al. Cancer statistics, 2006. CA Cancer J Clin. 2006;56:106-30.

2. Wils JA, Klein HO, Wagener DJT, Bleiberg H, Reis H, Korsten $\mathrm{F}$, et al. Sequential high-dose methotrexate and fluorouracil combined with doxorubicin--a step ahead in the treatment of advanced gastric cancer: a trial of the European Organization for Research and Treatment of Cancer Gastrointestinal Tract Cooperative Group. J Clin Oncol. 1991;9:827-31.

3. Vanhoefer U, Rougier P, Wilke H, Ducreux MP, Lacave AJ, Van Cutsem E, et al. Final results of a randomized phase III trial of sequential high-dose methotrexate, fluorouracil, and doxorubicin versus infusional fluorouracil and cisplatin in advanced gastric cancer: a trial of the European Organization for Research and Treatment of Cancer Gastrointestinal Tract Cooperative Group. J Clin Oncol. 2000;18:2648-57.

4. Ohtsu A, Shimada Y, Shirao K, Boku N, Hyodo I, Saito H, et al. Randomized phase III trial of fluorouracil alone versus fluorouracil plus cisplatin versus uracil and tegafur plus mitomycin in patients with advanced gastric cancer: JCOG study 9205. J Clin Oncol. 2003;21:54-9.

5. Webb A, Cunningham D, Scarffe JH, Harper P, Norman A, Joffe $\mathrm{JK}$, et al. Randomized trial comparing epirubicin, cisplatin, and fluorouracil versus fluorouracil, doxorubicin, and methotrexate in advanced esophagogastric cancer. J Clin Oncol. 1997;15:261-7.

6. Dank M, Zaluski J, Barone C, Valvere V, Yalcin S, Peschel C, et al. Randomized phase III study comparing irinotecan combined with 5-fluorouracil and folinic acid to cisplatin combined with 5-fluorouracil in chemotherapy naive patients with advanced adenocarcinoma of the stomach or esophagogastric junction. Ann Oncol. 2008;19:1450-7.
7. Cunningham D, Starling N, Rao S, Iveson T, Nicolson M, Coxon F, et al. Capecitabine and oxaliplatin for advanced esophagogastric cancer. N Engl J Med. 2008;358:36-46.

8. Van Cutsem E, Moiseyenko VM, Tjulandin S, Majlis A, Constenla M, Boni C, et al. Phase III study of docetaxel and cisplatin plus fluorouracil compared with cisplatin and fluorouracil as firstline therapy for advanced gastric cancer: a report of the V325 Study Group. J Clin Oncol. 2006;24:4991-7.

9. Ajani JA, Rodriguez W, Bodoky G, Moiseyenko CM, Lichinitser M, Gorbunova V, et al. Multicenter phase III comparison of cisplatin/S-1 with cisplatin/infusional fluorouracil in advanced gastric or gastroesophageal adenocarcinoma study: the FLAGS trial. J Clin Oncol. 2010;28:1547-53.

10. Boku N, Yamamoto S, Fukuda H, Shirao K, Doi T, Sawaki A, et al. Fluorouracil versus combination of irinotecan plus cisplatin versus S-1 in metastatic gastric cancer: a randomized phase 3 study. Lancet Oncol. 2009;10:1063-9.

11. Koizumi W, Narahara H, Hara T, Takagane A, Akiya T, Takagi $\mathrm{M}$, et al. Randomized phase III study of S-1 alone versus $\mathrm{S}-1+$ cisplatin in the treatment for advanced gastric cancer (The SPIRITS trial) SPIRITS: S-1 plus cisplatin vs S-1 in RCT in the treatment for stomach cancer. Lancet Oncol. 2008;9:215-21.

12. Narahara $H$, Iishi $H$, Tuburaya $A$, Chin $K$, Imamoto $H$, Esaki $T$, et al. Randomized phase III study comparing the efficacy and safety of irinotecan plus S-1 with S-1 alone as first-line treatment for advanced gastric cancer (study GC0301/TOP002). Gastric Cancer. 2011;14:72-80.

13. Wesolowski R, Lee C, Kim R. Is there a role for second-line chemotherapy in advanced gastric cancer? Lancet Oncol. 2009; 10:903-12.

14. Wilson D, Hiller L, Geh JI. Review of second-line chemotherapy for advanced gastric adenocarcinoma. Clin Oncol (R Coll Radiol). 2005;17:81-90.

15. Thuss-Patience PC, Kretzschmar A, Deist T, Hinke A, Bichev D, Lebedinzew B, et al. Irinotecan versus best supportive care (BSC) as second-line therapy in gastric cancer: a randomized phase III study of the Arbeitsgemeinschaft Internistische Onkologie (AIO) (abstract no. 4540). J Clin Oncol. 2009;27(Suppl 15S):4540.

16. Takashima A, Boku N, Kato K, Mizusawa J, Nakamura K, Fukuda $\mathrm{H}$, et al. Survival prolongation after treatment failure in patients with advanced gastric cancer (AGC): results from combined analysis of JCOG9205 and JCOG9912 (abstract no. 4061). J Clin Oncol. 2010;28(Suppl 15S):4061.

17. Bang YJ, Van Cutsem E, Feyereislova A, Chung HC, Shen L, Sawaki A, et al. Trastuzumab in combination with chemotherapy versus chemotherapy alone for treatment of HER2-positive advanced gastric or gastro-oesophageal junction cancer (ToGA): a phase 3, open-label, randomised controlled trial. Lancet. 2010;376:687-97.

18. Kang Y, Ohtsu A, Van Cutsem E, Rha SY, Sawaki A, Park S, et al. AVAGAST: a randomized, double-blind, placebo-controlled, phase III study of first-line capecitabine and cisplatin plus bevacizumab or placebo in patients with advanced gastric cancer (AGC). J Clin Oncol 2010; 28(Suppl 15S): LBA4007.

19. Kanagavel D, Pokataev IA, Fedyanin MY, Tryakin AA, Bazin IS, Narimanov MNA, et al. Prognostic model in patients treated for metastatic gastric cancer with second-line chemotherapy. Ann Oncol. 2010;21(9):1779-85.

20. Futatsuki K, Wakui A, Nakao I, Sakata Y, Kambe M, Shimada Y, et al. Late phase II study of irinotecan hydrochloride (CPT-11) in advanced gastric cancer. CPT-11 Gastrointestinal Cancer Study Group. Gan To Kagaku Ryoho. 1994;21:1033-8.

21. Yamada Y, Shirao K, Ohtsu A, Boku N, Hyodo I, Saitoh H, et al. Phase II trial of paclitaxel by three-hour infusion for advanced gastric cancer with short premedication for prophylaxis against paclitaxel-associated hypersensitivity reactions. Ann Oncol. 2001;12:1133-7. 
22. Koizumi W, Akiya T, Sato A, Yamaguchi K, Sakuyama T, Nakayama N, et al. Second-line chemotherapy with biweekly paclitaxel after failure of fluoropyrimidine-based treatment in patients with advanced or recurrent gastric cancer: a report from the Gastrointestinal Oncology Group of the Tokyo Cooperative Oncology Group, TCOG GC-0501 trial. Jpn J Clin Oncol. 2009;39:713-9.

23. Matsuda G, Kunisaki C, Makino H, Fukahori M, Kimura J, Sato $\mathrm{T}$, et al. Phase II study of weekly paclitaxel as a second-line treatment for S-1-refractory advanced gastric cancer. Anticancer Res. 2009;29:2863-7.

24. Taguchi T, Sakata Y, Kanamaru R, Kurihara M, Suminaga M, Ota J, et al. Late phase II clinical study of RP56976 (docetaxel) in patients with advanced/recurrent gastric cancer: a Japanese Cooperative Study Group trial (group A). Gan To Kagaku Ryoho. 1998;25:1915-24.

25. Mai M, Sakata Y, Kanamaru R, Kurihara M, Suminaga M, Ota J, et al. A late phase II clinical study of RP56976 (docetaxel) in patients with advanced or recurrent gastric cancer: a Cooperative Study Group Trial (group B). Gan To Kagaku Ryoho. 1999;26: 487-96.

26. Takiuchi $\mathrm{H}$, Goto $\mathrm{M}$, Imamura $\mathrm{H}$, Furukawa $\mathrm{H}$, Imano $\mathrm{M}$, Imamoto $\mathrm{H}$, et al. Multi-center phase II study for combination therapy with paclitaxel/doxifluridine to treat advanced/recurrent gastric cancer showing resistance to S-1 (OGSG 0302). Jpn J Clin Oncol. 2008;38:176-81.

27. Baize N, Abakar-Mahamat A, Mounier N, Berthier F, CaroliBosc FX. Phase II study of paclitaxel combined with capecitabine as second-line treatment for advanced gastric carcinoma after failure of cisplatin-based regimens. Cancer Chemother Pharmacol. 2009;64:549-55.

28. Yoshikawa T, Tsuburaya A, Shimada K, Sato A, Takahashi M, Koizumi W, et al. A phase II study of doxifluridine and docetaxel combination chemotherapy for advanced or recurrent gastric cancer. Gastric Cancer. 2009;12:212-8.

29. Sym SJ, Chang HM, Kang HJ, Lee SS, Ryu MH, Lee JL, et al. A phase II study of irinotecan and docetaxel combination chemotherapy for patients with previously treated metastatic or recurrent advanced gastric cancer. Cancer Chemother Pharmacol. 2008;63:1-8.

30. Barone C, Basso M, Schinzari G, Pozzo C, Trigila N, D’Argento $\mathrm{E}$, et al. Docetaxel and oxaliplatin combination in second-line treatment of patients with advanced gastric cancer. Gastric Cancer. 2007;10:104-11.

31. Kim SH, Lee GW, Go SI, Cho SH, Kim HJ, Kim HG, Kang JH. A phase II study of irinotecan, continuous 5-fluorouracil, and leucovorin (FOLFIRI) combination chemotherapy for patients with recurrent or metastatic gastric cancer previously treated with a fluoropyrimidine-based regimen. Am J Clin Oncol. 2010;33:572-6.

32. Leary A, Assersohn L, Cunningham D, Norman AR, Chong G, Brown G, et al. A phase II trial evaluating capecitabine and irinotecan as second line treatment in patients with oesophagogastric cancer who have progressed on, or within 3 months of platinum-based chemotherapy. Cancer Chemother Pharmacol. 2009;64:455-62.

33. Hamaguchi T, Shirao K, Yamamichi N, Hyodo I, Koizumi W, Seki S, et al. A phase II study of sequential methotrexate and 5-fluorouracil chemotherapy in previously treated gastric cancer: a report from the Gastrointestinal Oncology Group of the Japan Clinical Oncology Group, JCOG 9207 trial. Jpn J Clin Oncol. 2008;38:432-7.

34. Doi T, Muro K, Boku N, Yamada Y, Nishina T, Takiuchi H, et al. Multicenter phase II study of everolimus in patients with previously treated metastatic gastric cancer. J Clin Oncol. 2010;28: 1904-10. 